\title{
Supervised Feature Selection via Dependence Estimation
}

Le Song

LESONG@IT.USYD.EDU.AU

NICTA, Statistical Machine Learning Program, Canberra, ACT 0200, Australia; and University of Sydney

Alex Smola

ALEX.SMOLA@GMAIL.COM

NICTA, Statistical Machine Learning Program, Canberra, ACT 0200, Australia; and ANU

Arthur Gretton

ARTHUR.GRETTON@TUEBINGEN.MPG.DE

MPI for Biological Cybernetics, Spemannstr. 38, 72076 Tübingen, Germany

Karsten M. Borgwardt

BORGWARDT@DBS.IFI.LMU.DE

LMU, Department "Institute for Computer Science", Oettingenstr. 67, 80538 München, Germany

Justin Bedo

BEDO@IEEE.ORG

NICTA, Statistical Machine Learning Program, Canberra, ACT 0200, Australia

\begin{abstract}
We introduce a framework for filtering features that employs the Hilbert-Schmidt Independence Criterion (HSIC) as a measure of dependence between the features and the labels. The key idea is that good features should maximise such dependence. Feature selection for various supervised learning problems (including classification and regression) is unified under this framework, and the solutions can be approximated using a backward-elimination algorithm. We demonstrate the usefulness of our method on both artificial and real world datasets.
\end{abstract}

\section{Introduction}

In supervised learning problems, we are typically given $m$ data points $x \in \mathcal{X}$ and their labels $y \in \mathcal{Y}$. The task is to find a functional dependence between $x$ and $y, f: x \longmapsto y$, subject to certain optimality conditions. Representative tasks include binary classification, multi-class classification, regression and ranking. We often want to reduce the dimension of the data (the number of features) before the actual learning (Guyon \& Elisseeff, 2003); a larger number of features can be associated with higher data collection cost, more difficulty in model interpretation, higher computational cost for the classifier, and decreased generalisation

Appearing in Proceedings of the $24^{\text {th }}$ International Conference on Machine Learning, Corvallis, OR, 2007. Copyright 2007 by the author(s)/owner(s). ability. It is therefore important to select an informative feature subset.

The problem of supervised feature selection can be cast as a combinatorial optimisation problem. We have a full set of features, denoted $\mathcal{S}$ (whose elements correspond to the dimensions of the data). We use these features to predict a particular outcome, for instance the presence of cancer: clearly, only a subset $\mathcal{T}$ of features will be relevant. Suppose the relevance of $\mathcal{T}$ to the outcome is quantified by $\mathcal{Q}(\mathcal{T})$, and is computed by restricting the data to the dimensions in $\mathcal{T}$. Feature selection can then be formulated as

$$
\mathcal{T}_{0}=\arg \max _{\mathcal{T} \subseteq \mathcal{S}} \mathcal{Q}(\mathcal{T}) \quad \text { subject to } \quad|\mathcal{T}| \leq t,
$$

where $|\cdot|$ computes the cardinality of a set and $t$ upper bounds the number of selected features. Two important aspects of problem (1) are the choice of the criterion $\mathcal{Q}(\mathcal{T})$ and the selection algorithm.

Feature Selection Criterion. The choice of $\mathcal{Q}(\mathcal{T})$ should respect the underlying supervised learning tasks - estimate dependence function $f$ from training data and guarantee $f$ predicts well on test data. Therefore, good criteria should satisfy two conditions:

I: $\mathcal{Q}(\mathcal{T})$ is capable of detecting any desired (nonlinear as well as linear) functional dependence between the data and labels.

II: $\mathcal{Q}(\mathcal{T})$ is concentrated with respect to the underlying measure. This guarantees with high probability that the detected functional dependence is preserved in the test data. 
While many feature selection criteria have been explored, few take these two conditions explicitly into account. Examples include the leave-one-out error bound of SVM (Weston et al., 2000) and the mutual information (Koller \& Sahami, 1996). Although the latter has good theoretical justification, it requires density estimation, which is problematic for high dimensional and continuous variables. We sidestep these problems by employing a mutual-information like quantity - the Hilbert Schmidt Independence Criterion (HSIC) (Gretton et al., 2005). HSIC uses kernels for measuring dependence and does not require density estimation. HSIC also has good uniform convergence guarantees. As we show in section 2, HSIC satisfies conditions I and II, required for $\mathcal{Q}(\mathcal{T})$.

Feature Selection Algorithm. Finding a global optimum for (1) is in general NP-hard (Weston et al., 2003). Many algorithms transform (1) into a continuous problem by introducing weights on the dimensions (Weston et al., 2000, 2003). These methods perform well for linearly separable problems. For nonlinear problems, however, the optimisation usually becomes non-convex and a local optimum does not necessarily provide good features. Greedy approaches - forward selection and backward elimination - are often used to tackle problem (1) directly. Forward selection tries to increase $\mathcal{Q}(\mathcal{T})$ as much as possible for each inclusion of features, and backward elimination tries to achieve this for each deletion of features (Guyon et al., 2002). Although forward selection is computationally more efficient, backward elimination provides better features in general since the features are assessed within the context of all others.

BAHSIC. In principle, HSIC can be employed using either the forwards or backwards strategy, or a mix of strategies. However, in this paper, we will focus on a backward elimination algorithm. Our experiments show that backward elimination outperforms forward selection for HSIC. Backward elimination using HSIC (BAHSIC) is a filter method for feature selection. It selects features independent of a particular classifier. Such decoupling not only facilitates subsequent feature interpretation but also speeds up the computation over wrapper and embedded methods.

Furthermore, BAHSIC is directly applicable to binary, multiclass, and regression problems. Most other feature selection methods are only formulated either for binary classification or regression. The multi-class extension of these methods is usually accomplished using a one-versus-the-rest strategy. Still fewer methods handle classification and regression cases at the same time. BAHSIC, on the other hand, accommodates all these cases in a principled way: by choosing different kernels, BAHSIC also subsumes many existing methods as special cases. The versatility of BAHSIC originates from the generality of HSIC. Therefore, we begin our exposition with an introduction of HSIC.

\section{Measures of Dependence}

We define $\mathcal{X}$ and $\mathcal{Y}$ broadly as two domains from which we draw samples $(x, y)$ : these may be real valued, vector valued, class labels, strings, graphs, and so on. We define a (possibly nonlinear) mapping $\phi(x) \in \mathcal{F}$ from each $x \in \mathcal{X}$ to a feature space $\mathcal{F}$, such that the inner product between the features is given by a kernel function $k\left(x, x^{\prime}\right):=\left\langle\phi(x), \phi\left(x^{\prime}\right)\right\rangle: \mathcal{F}$ is called a reproducing kernel Hilbert space (RKHS). Likewise, let $\mathcal{G}$ be a second RKHS on $\mathcal{Y}$ with kernel $l(\cdot, \cdot)$ and feature map $\psi(y)$. We may now define a cross-covariance operator between these feature maps, in accordance with Baker (1973); Fukumizu et al. (2004): this is a linear operator $\mathcal{C}_{x y}: \mathcal{G} \longmapsto \mathcal{F}$ such that

$$
\mathcal{C}_{x y}=\mathbb{E}_{x y}\left[\left(\phi(x)-\mu_{x}\right) \otimes\left(\psi(y)-\mu_{y}\right)\right],
$$

where $\otimes$ is the tensor product. The square of the Hilbert-Schmidt norm of the cross-covariance operator (HSIC), $\left\|\mathcal{C}_{x y}\right\|_{\mathrm{HS}}^{2}$, is then used as our feature selection criterion $\mathcal{Q}(\mathcal{T})$. Gretton et al. (2005) show that HSIC can be expressed in terms of kernels as

$$
\begin{aligned}
& \operatorname{HSIC}(\mathcal{F}, \mathcal{G}, \underset{x y}{\operatorname{Pr}})=\left\|\mathcal{C}_{x y}\right\|_{\mathrm{HS}}^{2} \\
& =\mathbb{E}_{x x^{\prime} y y^{\prime}}\left[k\left(x, x^{\prime}\right) l\left(y, y^{\prime}\right)\right]+\mathbb{E}_{x x^{\prime}}\left[k\left(x, x^{\prime}\right)\right] \mathbb{E}_{y y^{\prime}}\left[l\left(y, y^{\prime}\right)\right] \\
& -2 \mathbb{E}_{x y}\left[\mathbb{E}_{x^{\prime}}\left[k\left(x, x^{\prime}\right)\right] \mathbb{E}_{y^{\prime}}\left[l\left(y, y^{\prime}\right)\right]\right],
\end{aligned}
$$

where $\mathrm{E}_{x x^{\prime} y y^{\prime}}$ is the expectation over both $(x, y) \sim$ $\operatorname{Pr}_{x y}$ and an additional pair of variables $\left(x^{\prime}, y^{\prime}\right) \sim \operatorname{Pr}_{x y}$ drawn independently according to the same law. Previous work used HSIC to measure independence between two sets of random variables (Gretton et al., 2005). Here we use it to select a subset $\mathcal{T}$ from the first full set of random variables $\mathcal{S}$. We now describe further properties of HSIC which support its use as a feature selection criterion.

Property (I) Gretton et al. (2005, Theorem 4) show that whenever $\mathcal{F}, \mathcal{G}$ are RKHSs with universal kernels $k, l$ on respective compact domains $\mathcal{X}$ and $\mathcal{Y}$ in the sense of Steinwart $(2002)$, then $\operatorname{HSIC}\left(\mathcal{F}, \mathcal{G}, \operatorname{Pr}_{x y}\right)=0$ if and only if $x$ and $y$ are independent. In terms of feature selection, a universal kernel such as the Gaussian RBF kernel or the Laplace kernel permits HSIC to detect any dependence between $\mathcal{X}$ and $\mathcal{Y}$. HSIC is zero if and only if features and labels are independent.

In fact, non-universal kernels can also be used for HSIC, although they may not guarantee that all de- 
pendencies are detected. Different kernels incorporate distinctive prior knowledge into the dependence estimation, and they focus HSIC on dependence of a certain type. For instance, a linear kernel requires HSIC to seek only second order dependence. Clearly HSIC is capable of finding and exploiting dependence of a much more general nature by kernels on graphs, strings, or other discrete domains.

Property (II) Given a sample $Z=$ $\left\{\left(x_{1}, y_{1}\right), \ldots,\left(x_{m}, y_{m}\right)\right\}$ of size $m$ drawn from $\operatorname{Pr}_{x y}$, we derive an unbiased estimate of HSIC,

$$
\begin{aligned}
& \operatorname{HSIC}(\mathcal{F}, \mathcal{G}, Z) \\
& =\frac{1}{m(m-3)}\left[\operatorname{tr}(\mathbf{K L})+\frac{\mathbf{1}^{\top} \mathbf{K} \mathbf{1} \mathbf{1}^{\top} \mathbf{L} \mathbf{1}}{(m-1)(m-2)}-\frac{2}{m-2} \mathbf{1}^{\top} \mathbf{K} \mathbf{L} \mathbf{1}\right],
\end{aligned}
$$

where $\mathbf{K}$ and $\mathbf{L}$ are computed as $\mathbf{K}_{i j}=$ $\left(1-\delta_{i j}\right) k\left(x_{i}, x_{j}\right)$ and $\mathbf{L}_{i j}=\left(1-\delta_{i j}\right) l\left(y_{i}, y_{j}\right)$. Note that the diagonal entries of $\mathbf{K}$ and $\mathbf{L}$ are set to zero. The following theorem, a formal statement that the empirical HSIC is unbiased, is proved in (Song et al., 2007).

Theorem 1 (HSIC is Unbiased) Let $\mathbb{E}_{Z}$ denote the expectation taken over $m$ independent observations $\left(x_{i}, y_{i}\right)$ drawn from $\operatorname{Pr}_{x y}$. Then

$$
\operatorname{HSIC}(\mathcal{F}, \mathcal{G}, \underset{x y}{\operatorname{Pr}})=\mathbb{E}_{Z}[\operatorname{HSIC}(\mathcal{F}, \mathcal{G}, Z)] .
$$

This property is by contrast with the mutual information, which can require sophisticated bias correction strategies (e.g. Nemenman et al., 2002).

U-Statistics. The estimator in (4) can be alternatively formulated using U-statistics,

$$
\operatorname{HSIC}(\mathcal{F}, \mathcal{G}, Z)=(m)_{4}^{-1} \sum_{(i, j, q, r) \in \mathbf{i}_{4}^{m}}^{m} h(i, j, q, r),
$$

where $(m)_{n}=\frac{m !}{(m-n) !}$ is the Pochhammer coefficient and where $\mathbf{i}_{r}^{m}$ denotes the set of all $r$-tuples drawn without replacement from $\{1, \ldots, m\}$. The kernel $h$ of the U-statistic is defined by

$$
\frac{1}{4 !} \sum_{(s, t, u, v)}^{(i, j, q, r)}\left(\mathbf{K}_{s t} \mathbf{L}_{s t}+\mathbf{K}_{s t} \mathbf{L}_{u v}-2 \mathbf{K}_{s t} \mathbf{L}_{s u}\right),
$$

where the sum in (7) represents all ordered quadruples $(s, t, u, v)$ selected without replacement from $(i, j, q, r)$.

We now show that $\operatorname{HSIC}(\mathcal{F}, \mathcal{G}, Z)$ is concentrated. Furthermore, its convergence in probability to $\operatorname{HSIC}\left(\mathcal{F}, \mathcal{G}, \operatorname{Pr}_{x y}\right)$ occurs with rate $1 / \sqrt{m}$ which is a slight improvement over the convergence of the biased estimator by Gretton et al. (2005).
Theorem 2 (HSIC is Concentrated) Assume $k, l$ are bounded almost everywhere by 1 , and are nonnegative. Then for $m>1$ and all $\delta>0$, with probability at least $1-\delta$ for all $\operatorname{Pr}_{x y}$

$$
|\operatorname{HSIC}(\mathcal{F}, \mathcal{G}, Z)-\operatorname{HSIC}(\mathcal{F}, \mathcal{G}, \underset{x y}{\operatorname{Pr}})| \leq 8 \sqrt{\log (2 / \delta) / m}
$$

By virtue of (6) we see immediately that HSIC is a U-statistic of order 4, where each term is bounded in $[-2,2]$. Applying Hoeffding's bound as in Gretton et al. (2005) proves the result.

These two theorems imply the empirical HSIC closely reflects its population counterpart. This means the same features should consistently be selected to achieve high dependence if the data are repeatedly drawn from the same distribution.

Asymptotic Normality. It follows from Serfling (1980) that under the assumptions $\mathbb{E}\left(h^{2}\right)<\infty$ and that the data and labels are not independent, the empirical HSIC converges in distribution to a Gaussian random variable with mean $\operatorname{HSIC}\left(\mathcal{F}, \mathcal{G}, \operatorname{Pr}_{x y}\right)$ and variance

$$
\begin{aligned}
\sigma_{\mathrm{HSIC}}^{2} & =\frac{16}{m}\left(R-\mathrm{HSIC}^{2}\right), \text { where } \\
R & =\frac{1}{m} \sum_{i=1}^{m}\left((m-1)_{3}^{-1} \sum_{(j, q, r) \in \mathbf{i}_{3}^{m} \backslash\{i\}} h(i, j, q, r)\right)^{2},
\end{aligned}
$$

and $\mathbf{i}_{r}^{m} \backslash\{i\}$ denotes the set of all $r$-tuples drawn without replacement from $\{1, \ldots, m\} \backslash\{i\}$. The asymptotic normality allows us to formulate statistics for a significance test. This is useful because it may provide an assessment of the dependence between the selected features and the labels.

Simple Computation. Note that $\operatorname{HSIC}(\mathcal{F}, \mathcal{G}, Z)$ is simple to compute, since only the kernel matrices $\mathbf{K}$ and $\mathbf{L}$ are needed, and no density estimation is involved. For feature selection, $\mathbf{L}$ is fixed through the whole process. It can be precomputed and stored for speedup if needed. Note also that $\operatorname{HSIC}(\mathcal{F}, \mathcal{G}, Z)$ does not need any explicit regularisation parameter. This is encapsulated in the choice of the kernels.

\section{Feature Selection via HSIC}

Having defined our feature selection criterion, we now describe an algorithm that conducts feature selection on the basis of this dependence measure. Using HSIC, we can perform both backward (BAHSIC) and forward (FOHSIC) selection of the features. In particular, when we use a linear kernel on the data (there is no such requirement for the labels), forward selection 
and backward selection are equivalent: the objective function decomposes into individual coordinates, and thus feature selection can be done without recursion in one go. Although forward selection is computationally more efficient, backward elimination in general yields better features, since the quality of the features is assessed within the context of all other features. Hence we present the backward elimination version of our algorithm here (a forward greedy selection version can be derived similarly).

BAHSIC appends the features from $\mathcal{S}$ to the end of a list $\mathcal{S}^{\dagger}$ so that the elements towards the end of $\mathcal{S}^{\dagger}$ have higher relevance to the learning task. The feature selection problem in (1) can be solved by simply taking the last $t$ elements from $\mathcal{S}^{\dagger}$. Our algorithm produces $\mathcal{S}^{\dagger}$ recursively, eliminating the least relevant features from $\mathcal{S}$ and adding them to the end of $\mathcal{S}^{\dagger}$ at each iteration. For convenience, we also denote HSIC as $\operatorname{HSIC}(\sigma, \mathcal{S})$, where $\mathcal{S}$ are the features used in computing the data kernel matrix $\mathbf{K}$, and $\sigma$ is the parameter for the data kernel (for instance, this might be the size of a Gaussian kernel $\left.k\left(x, x^{\prime}\right)=\exp \left(-\sigma\left\|x-x^{\prime}\right\|^{2}\right)\right)$.

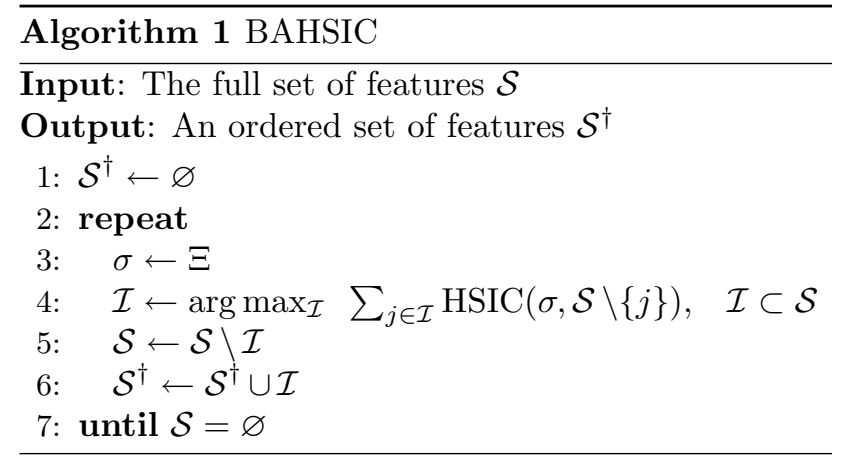

Step 3 of the algorithm denotes a policy for adapting the kernel parameters, e.g. by optimising over the possible parameter choices. In our experiments, we typically normalize each feature separately to zero mean and unit variance, and adapt the parameter for a Gaussian kernel by setting $\sigma$ to $1 /(2 d)$, where $d=|\mathcal{S}|-1$. If we have prior knowledge about the type of nonlinearity, we can use a kernel with fixed parameters for BAHSIC. In this case, step 3 can be omitted.

Step 4 of the algorithm is concerned with the selection of a set $\mathcal{I}$ of features to eliminate. While one could choose a single element of $\mathcal{S}$, this would be inefficient when there are a large number of irrelevant features. On the other hand, removing too many features at once risks the loss of relevant features. In our experiments, we found a good compromise between speed and feature quality was to remove $10 \%$ of the current features at each iteration.

\section{Connections to Other Approaches}

We now explore connections to other feature selectors. For binary classification, an alternative criterion for selecting features is to check whether the distributions $\operatorname{Pr}(x \mid y=1)$ and $\operatorname{Pr}(x \mid y=-1)$ differ. For this purpose one could use Maximum Mean Discrepancy (MMD) (Borgwardt et al., 2006). Likewise, one could use Kernel Target Alignment (KTA) (Cristianini et al., 2003) to test directly whether there exists any correlation between data and labels. KTA has been used for feature selection. Formally it is defined as $\operatorname{tr} \mathbf{K} \mathbf{L} /\|\mathbf{K}\|\|\mathbf{L}\|$. For computational convenience the normalisation is often omitted in practise (Neumann et al., 2005), which leaves us with $\operatorname{tr} \mathbf{K} \mathbf{L}$. We discuss this unnormalised variant below.

Let us consider the output kernel $l\left(y, y^{\prime}\right)=\rho(y) \rho\left(y^{\prime}\right)$, where $\rho(1)=m_{+}^{-1}$ and $\rho(-1)=-m_{-}^{-1}$, and $m_{+}$and $m_{-}$are the numbers of positive and negative samples, respectively. With this kernel choice, we show that MMD and KTA are closely related to HSIC. The following theorem is proved in the appendix.

\section{Theorem 3 (Connection to MMD and KTA)}

Assume the kernel $k\left(x, x^{\prime}\right)$ for the data is bounded and the kernel for the labels is $l\left(y, y^{\prime}\right)=\rho(y) \rho\left(y^{\prime}\right)$. Then

$$
\begin{aligned}
\left|\mathrm{HSIC}-(m-1)^{-2} \mathrm{MMD}\right| & =O\left(m^{-1}\right) \\
\left|\mathrm{HSIC}-(m-1)^{-2} \mathrm{KTA}\right| & =O\left(m^{-1}\right) .
\end{aligned}
$$

This means selecting features that maximise HSIC also maximises MMD and KTA. Note that in general (multiclass, regression, or generic binary classification) this connection does not hold.

\section{Variants of BAHSIC}

New variants can be readily derived from BAHSIC by combining the two building blocks of BAHSIC: a kernel on the data and another one on the labels. Here we provide three examples using a Gaussian kernel on the data, while varying the kernel on the labels. This provides us with feature selectors for three problems:

Binary classification (BIN) We set $m_{+}^{-1}$ as the label for positive class members, and $m_{-}^{-1}$ for negative class members. We then apply a linear kernel.

Multiclass classification (MUL) We apply a linear kernel on the labels using the label vectors below, as described for a 3 -class example. Here $m_{i}$ is the number 
of samples in class $i$ and $\mathbf{1}_{m_{i}}$ denotes a vector of all ones with length $m_{i}$.

$$
\mathbf{Y}=\left(\begin{array}{ccc}
\frac{\mathbf{1}_{m_{1}}}{m_{1}} & \frac{\mathbf{1}_{m_{1}}}{m_{2}-m} & \frac{\mathbf{1}_{m_{1}}}{m_{3}-m} \\
\frac{\mathbf{1}_{m_{2}}}{m_{1}-m} & \frac{\mathbf{1}_{m_{2}}}{m_{2}} & \frac{\mathbf{1}_{m_{2}}}{m_{3}-m} \\
\frac{\mathbf{1}_{m_{3}}}{m_{1}-m} & \frac{\mathbf{1}_{m_{3}}}{m_{2}-m} & \frac{\mathbf{1}_{m_{3}}}{m_{3}}
\end{array}\right)_{m \times 3} .
$$

Regression (REG) A Gaussian RBF kernel is also used on the labels. For convenience the kernel width $\sigma$ is fixed as the median distance between points in the sample (Schölkopf \& Smola, 2002).

For the above variants a further speedup of BAHSIC is possible by updating the entries of the kernel matrix incrementally, since we are using an RBF kernel. We use the fact that $\left\|x-x^{\prime}\right\|^{2}=\sum_{j}\left\|x_{j}-x_{j}^{\prime}\right\|^{2}$. Hence $\left\|x-x^{\prime}\right\|^{2}$ needs to be computed only once. Subsequent updates are effected by subtracting $\left\|x_{j}-x_{j}^{\prime}\right\|^{2}$ (subscript here indices dimension).

We will use BIN, MUL and REG as the particular instances of BAHSIC in our experiments. We will refer to them commonly as BAHSIC since the exact meaning will be clear depending on the datasets encountered. Furthermore, we also instantiate FOHSIC using the same kernels as BIN, MUL and REG, and we adopt the same convention when we refer to it in our experiments.

\section{Experimental Results}

We conducted three sets of experiments. The characteristics of the datasets and the aims of the experiments are: $(i)$ artificial datasets illustrating the properties of BAHSIC; (ii) real datasets that compare BAHSIC with other methods; and (iii) a brain computer interface dataset showing that BAHSIC selects meaningful features.

\subsection{Artificial datasets}

We constructed 3 artificial datasets, as illustrated in Figure 1, to illustrate the difference between BAHSIC variants with linear and nonlinear kernels. Each dataset has 22 dimensions - only the first two dimensions are related to the prediction task and the rest are just Gaussian noise. These datasets are $(i)$ Binary XOR data: samples belonging to the same class have multimodal distributions; (ii) Multiclass data: there are 4 classes but 3 of them are collinear; (iii) Nonlinear regression data: labels are related to the first two dimension of the data by $y=x_{1} \exp \left(-x_{1}^{2}-x_{2}^{2}\right)+\epsilon$, where $\epsilon$ denotes additive Gaussian noise. We compare BAHSIC to FOHSIC, Pearson's correlation, mutual information (Zaffalon \& Hutter, 2002), and RELIEF (RELIEF works only for binary problems). We aim to show that when nonlinear dependencies exist in the
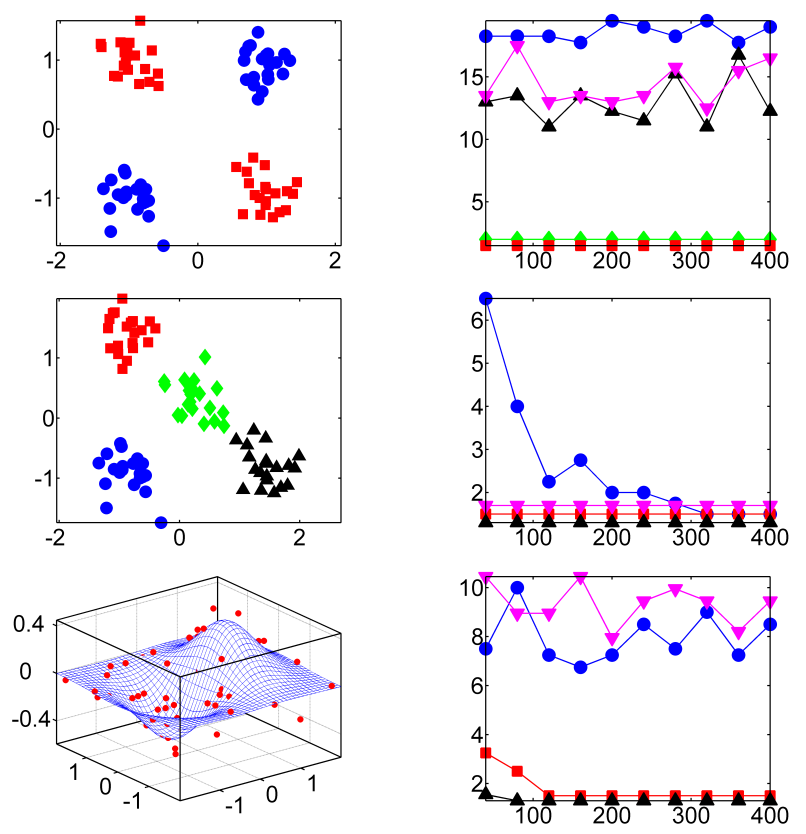

Figure 1: Artificial datasets and the performance of different methods when varying the number of observations. Left column, top to bottom: Binary, multiclass, and regression data. Different classes are encoded with different colours. Right column: Median rank (y-axis) of the two relevant features as a function of sample size ( $\mathrm{x}-$ axis) for the corresponding datasets in the left column. (Blue circle: Pearson's correlation; Green triangle: RELIEF; Magenta downward triangle: mutual information; Black triangle: FOHSIC; Red square: BAHSIC.)

data, BAHSIC with nonlinear kernels is very competent in finding them.

We instantiate the artificial datasets over a range of sample sizes (from 40 to 400), and plot the median rank, produced by various methods, for the first two dimensions of the data. All numbers in Figure 1 are averaged over 10 runs. In all cases, BAHSIC shows good performance. More specifically, we observe:

Binary XOR Both BAHSIC and RELIEF correctly select the first two dimensions of the data even for small sample sizes; while FOHSIC, Pearson's correlation, and mutual information fail. This is because the latter three evaluate the goodness of each feature independently. Hence they are unable to capture nonlinear interaction between features.

Multiclass Data BAHSIC, FOHSIC and mutual information select the correct features irrespective of the size of the sample. Pearson's correlation only works for large sample size. The collinearity of 3 classes provides linear correlation between the data and the labels, but due to the interference of the fourth class such corre- 
lation is picked up by Pearson's correlation only for a large sample size.

Nonlinear Regression Data The performance of Pearson's correlation and mutual information is slightly better than random. BAHSIC and FOHSIC quickly converge to the correct answer as the sample size increases.

In fact, we observe that as the sample size increases, BAHSIC is able to rank the relevant features (the first two dimensions) almost correctly in the first iteration (results not shown). While this does not prove BAHSIC with nonlinear kernels is always better than that with a linear kernel, it illustrates the competence of BAHSIC in detecting nonlinear features. This is obviously useful in a real-world situations. The second advantage of BAHSIC is that it is readily applicable to both classification and regression problems, by simply choosing a different kernel on the labels.

\subsection{Real world datasets}

Algorithms In this experiment, we show that the performance of BAHSIC can be comparable to other state-of-the-art feature selectors, namely SVM Recursive Feature Elimination (RFE) (Guyon et al.,

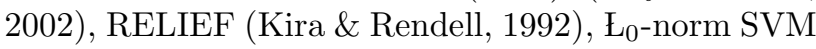
$\left(\mathrm{E}_{0}\right)$ (Weston et al., 2003), and R2W2 (Weston et al., 2000). We used the implementation of these algorithms as given in the Spider machine learning toolbox, since those were the only publicly available implementations. ${ }^{1}$ Furthermore, we also include filter methods, namely FOHSIC, Pearson's correlation (PC), and mutual information (MI), in our comparisons.

Datasets We used various real world datasets taken from the UCI repository, ${ }^{2}$ the Statlib repository, ${ }^{3}$ the LibSVM website, ${ }^{4}$ and the NIPS feature selection challenge ${ }^{5}$ for comparison. Due to scalability issues in Spider, we produced a balanced random sample of size less than 2000 for datasets with more than 2000 samples.

Experimental Protocol We report the performance of an SVM using a Gaussian kernel on a feature subset of size 5 and 10 -fold cross-validation. These 5 features were selected per fold using different methods. Since we are comparing the selected features, we used the same SVM for all methods: a Gaussian kernel with $\sigma$ set as the median distance between points in the sample (Schölkopf \& Smola, 2002) and regular-

\footnotetext{
${ }^{1}$ http://www.kyb.tuebingen.mpg.de/bs/people/spider

${ }^{2}$ http://www.ics.uci.edu/ mlearn/MLSummary.html

${ }^{3}$ http://lib.stat.cmu.edu/datasets/

${ }^{4}$ http://www.csie.ntu.edu.tw/ cjlin/libsvmtools/datasets/

${ }^{5}$ http://clopinet.com/isabelle/Projects/NIPS2003/
}

ization parameter $C=100$. On classification datasets, we measured the performance using the error rate, and on regression datasets we used the percentage of variance not-explained (also known as $1-r^{2}$ ). The results for binary datasets are summarized in the first part of Table 1. Those for multiclass and regression datasets are reported respectively in the second and the third parts of Table 1.

To provide a concise summary of the performance of various methods on binary datasets, we measured how the methods compare with the best performing one in each dataset in Table 1. We recorded the best absolute performance of all feature selectors as the baseline, and computed the distance of each algorithm to the best possible result. In this context it makes sense to penalize catastrophic failures more than small deviations. In other words, we would like to have a method which is at least almost always very close to the best performing one. Taking the $\ell_{2}$ distance achieves this effect, by penalizing larger differences more heavily. It is also our goal to choose an algorithm that performs homogeneously well across all datasets. The $\ell_{2}$ distance scores are listed for the binary datasets in Table 1. In general, the smaller the $\ell_{2}$ distance, the better the method. In this respect, BAHSIC and FOHSIC have the best performance. We did not produce the $\ell_{2}$ distance for multiclass and regression datasets, since the limited number of such datasets did not allow us to draw statistically significant conclusions.

\subsection{Brain-computer interface dataset}

In this experiment, we show that BAHSIC selects features that are meaningful in practise: we use BAHSIC to select a frequency band for a brain-computer interface (BCI) data set from the Berlin BCI group (Dornhege et al., 2004). The data contains EEG signals (118 channels, sampled at $100 \mathrm{~Hz}$ ) from five healthy subjects ('aa', 'al', 'av', 'aw' and 'ay') recorded during two types of motor imaginations. The task is to classify the imagination for individual trials.

Our experiment proceeded in 3 steps: (i) A Fast Fourier transformation (FFT) was performed on each channel and the power spectrum was computed. (ii) The power spectra from all channels were averaged to obtain a single spectrum for each trial. (iii) BAH-

Table 2: Classification errors (\%) on BCI data after selecting a frequency range.

\begin{tabular}{|c|c|c|c|c|c|}
\hline Subject & aa & al & av & aw & ay \\
\hline CSP & $17.5 \pm 2.5$ & $3.1 \pm 1.2$ & $32.1 \pm 2.5$ & $7.3 \pm 2.7$ & $\mathbf{6 . 0} \pm \mathbf{1 . 6}$ \\
CSSP & $14.9 \pm 2.9$ & $2.4 \pm 1.3$ & $33.0 \pm 2.7$ & $\mathbf{5 . 4} \pm \mathbf{1 . 9}$ & $6.2 \pm 1.5$ \\
CSSSP & $\mathbf{1 2 . 2} \pm \mathbf{2 . 1}$ & $2.2 \pm 0.9$ & $31.8 \pm 2.8$ & $6.3 \pm 1.8$ & $12.7 \pm 2.0$ \\
BAHSIC & $13.7 \pm 4.3$ & $\mathbf{1 . 9} \pm \mathbf{1 . 3}$ & $\mathbf{3 0 . 5} \pm \mathbf{3 . 3}$ & $6.1 \pm 3.8$ & $9.0 \pm 6.0$ \\
\hline
\end{tabular}


Table 1: Classification error (\%) or percentage of variance not-explained (\%). The best result, and those results not significantly worse than it, are highlighted in bold (one-sided Welch t-test with 95\% confidence level). 100.0 $\pm 0.0^{*}$ : program is not finished in a week or crashed. -: not applicable.

\begin{tabular}{|c|c|c|c|c|c|c|c|c|}
\hline Data & BAHSIC & FOHSIC & $\overline{\mathbf{P C}}$ & MI & RFE & RELIEF & $\mathrm{E}_{0}$ & R2W2 \\
\hline covertype & $26.3 \pm 1.5$ & $37.9 \pm 1.7$ & $40.3 \pm 1.3$ & $26.7 \pm 1.1$ & $33.0 \pm 1.9$ & $42.7 \pm 0.7$ & $43.4 \pm 0.7$ & $44.2 \pm 1.7$ \\
\hline ionosphere & $12.3 \pm 1.7$ & $12.8 \pm 1.6$ & $12.3 \pm 1.5$ & $13.1 \pm 1.7$ & $20.2 \pm 2.2$ & $11.7 \pm 2.0$ & $35.9 \pm 0.4$ & $13.7 \pm 2.7$ \\
\hline sonar & $27.9 \pm 3.1$ & $25.0 \pm 2.3$ & $25.5 \pm 2.4$ & $26.9 \pm 1.9$ & $21.6 \pm 3.4$ & $24.0 \pm 2.4$ & $36.5 \pm 3.3$ & $32.3 \pm 1.8$ \\
\hline heart & $14.8 \pm 2.4$ & $14.4 \pm 2.4$ & $16.7 \pm 2.4$ & $15.2 \pm 2.5$ & $21.9 \pm 3.0$ & $21.9 \pm 3.4$ & $30.7 \pm 2.8$ & $19.3 \pm 2.6$ \\
\hline breastcancer & $3.8 \pm 0.4$ & $3.8 \pm 0.4$ & $4.0 \pm 0.4$ & $3.5 \pm 0.5$ & $3.4 \pm 0.6$ & $3.1 \pm 0.3$ & $32.7 \pm 2.3$ & $3.4 \pm 0.4$ \\
\hline australian & $14.3 \pm 1.3$ & $14.3 \pm 1.3$ & $14.5 \pm 1.3$ & $14.5 \pm 1.3$ & $14.8 \pm 1.2$ & $14.5 \pm 1.3$ & $35.9 \pm 1.0$ & $14.5 \pm 1.3$ \\
\hline splice & $22.6 \pm 1.1$ & $22.6 \pm 1.1$ & $22.8 \pm 0.9$ & $21.9 \pm 1.0$ & $20.7 \pm 1.0$ & $22.3 \pm 1.0$ & $45.2 \pm 1.2$ & $24.0 \pm 1.0$ \\
\hline svmguide3 & $20.8 \pm 0.6$ & $20.9 \pm 0.6$ & $21.2 \pm 0.6$ & $20.4 \pm 0.7$ & $21.0 \pm 0.7$ & $21.6 \pm 0.4$ & $23.3 \pm 0.3$ & $23.9 \pm 0.2$ \\
\hline adult & $24.8 \pm 0.2$ & $24.4 \pm 0.6$ & $18.3 \pm 1.1$ & $21.6 \pm 1.1$ & $21.3 \pm 0.9$ & $24.4 \pm 0.2$ & $24.7 \pm 0.1$ & $100.0 \pm 0.0^{*}$ \\
\hline cleveland & $19.0 \pm 2.1$ & $20.5 \pm 1.9$ & $21.9 \pm 1.7$ & $19.5 \pm 2.2$ & $20.9 \pm 2.1$ & $22.4 \pm 2.5$ & $25.2 \pm 0.6$ & $21.5 \pm 1.3$ \\
\hline derm & $0.3 \pm 0.3$ & $0.3 \pm 0.3$ & $0.3 \pm 0.3$ & $0.3 \pm 0.3$ & $0.3 \pm 0.3$ & $0.3 \pm 0.3$ & $24.3 \pm 2.6$ & $0.3 \pm 0.3$ \\
\hline hepatitis & $13.8 \pm 3.5$ & $15.0 \pm 2.5$ & $15.0 \pm 4.1$ & $15.0 \pm 4.1$ & $15.0 \pm 2.5$ & $17.5 \pm 2.0$ & $16.3 \pm 1.9$ & $17.5 \pm 2.0$ \\
\hline musk & $29.9 \pm 2.5$ & $29.6 \pm 1.8$ & $26.9 \pm 2.0$ & $31.9 \pm 2.0$ & $34.7 \pm 2.5$ & $27.7 \pm 1.6$ & $42.6 \pm 2.2$ & $36.4 \pm 2.4$ \\
\hline optdigits & $0.5 \pm 0.2$ & $0.5 \pm 0.2$ & $0.5 \pm 0.2$ & $3.4 \pm 0.6$ & $3.0 \pm 1.6$ & $0.9 \pm 0.3$ & $12.5 \pm 1.7$ & $0.8 \pm 0.3$ \\
\hline specft & $20.0 \pm 2.8$ & $20.0 \pm 2.8$ & $18.8 \pm 3.4$ & $18.8 \pm 3.4$ & $37.5 \pm 6.7$ & $26.3 \pm 3.5$ & $36.3 \pm 4.4$ & $31.3 \pm 3.4$ \\
\hline wdbc & $5.3 \pm 0.6$ & $5.3 \pm 0.6$ & $5.3 \pm 0.7$ & $6.7 \pm 0.5$ & $7.7 \pm 1.8$ & $7.2 \pm 1.0$ & $16.7 \pm 2.7$ & $6.8 \pm 1.2$ \\
\hline wine & $1.7 \pm 1.1$ & $1.7 \pm 1.1$ & $1.7 \pm 1.1$ & $1.7 \pm 1.1$ & $3.4 \pm 1.4$ & $4.2 \pm 1.9$ & $25.1 \pm 7.2$ & $1.7 \pm 1.1$ \\
\hline german & $29.2 \pm 1.9$ & $29.2 \pm 1.8$ & $26.2 \pm 1.5$ & $26.2 \pm 1.7$ & $27.2 \pm 2.4$ & $33.2 \pm 1.1$ & $32.0 \pm 0.0$ & $24.8 \pm 1.4$ \\
\hline gisette & $12.4 \pm 1.0$ & $13.0 \pm 0.9$ & $16.0 \pm 0.7$ & $50.0 \pm 0.0$ & $42.8 \pm 1.3$ & $16.7 \pm 0.6$ & $42.7 \pm 0.7$ & $100.0 \pm 0.0^{*}$ \\
\hline arcene & $22.0 \pm 5.1$ & $19.0 \pm 3.1$ & $31.0 \pm 3.5$ & $45.0 \pm 2.7$ & $34.0 \pm 4.5$ & $30.0 \pm 3.9$ & $46.0 \pm 6.2$ & $32.0 \pm 5.5$ \\
\hline madelon & $37.9 \pm 0.8$ & $38.0 \pm 0.7$ & $38.4 \pm 0.6$ & $51.6 \pm 1.0$ & $41.5 \pm 0.8$ & $38.6 \pm 0.7$ & $51.3 \pm 1.1$ & $100.0 \pm 0.0^{*}$ \\
\hline$\ell_{2}$ & 11.2 & 14.8 & 19.7 & 48.6 & 42.2 & 25.9 & 85.0 & 138.3 \\
\hline satimage & $15.8 \pm 1.0$ & $17.9 \pm 0.8$ & $52.6 \pm 1.7$ & $22.7 \pm 0.9$ & $18.7 \pm 1.3$ & - & $22.1 \pm 1.8$ & - \\
\hline segment & $28.6 \pm 1.3$ & $33.9 \pm 0.9$ & $22.9 \pm 0.5$ & $27.1 \pm 1.3$ & $24.5 \pm 0.8$ & - & $68.7 \pm 7.1$ & - \\
\hline vehicle & $36.4 \pm 1.5$ & $48.7 \pm 2.2$ & $42.8 \pm 1.4$ & $45.8 \pm 2.5$ & $35.7 \pm 1.3$ & - & $40.7 \pm 1.4$ & - \\
\hline svmguide2 & $22.8 \pm 2.7$ & $22.2 \pm 2.8$ & $26.4 \pm 2.5$ & $27.4 \pm 1.6$ & $35.6 \pm 1.3$ & - & $34.5 \pm 1.7$ & - \\
\hline vowel & $44.7 \pm 2.0$ & $44.7 \pm 2.0$ & $48.1 \pm 2.0$ & $45.4 \pm 2.2$ & $51.9 \pm 2.0$ & - & $85.6 \pm 1.0$ & - \\
\hline usps & $43.4 \pm 1.3$ & $43.4 \pm 1.3$ & $73.7 \pm 2.2$ & $67.8 \pm 1.8$ & $55.8 \pm 2.6$ & - & $67.0 \pm 2.2$ & - \\
\hline housing & $18.5 \pm 2.6$ & $\overline{18.9 \pm 3.6}$ & $25.3 \pm 2.5$ & $\overline{18.9} \pm 2.7$ & - & - & - & - \\
\hline bodyfat & $3.5 \pm 2.5$ & $3.5 \pm 2.5$ & $3.4 \pm 2.5$ & $3.4 \pm 2.5$ & - & - & - & - \\
\hline abalone & $55.1 \pm 2.7$ & $55.9 \pm 2.9$ & $54.2 \pm 3.3$ & $56.5 \pm 2.6$ & - & - & - & - \\
\hline
\end{tabular}
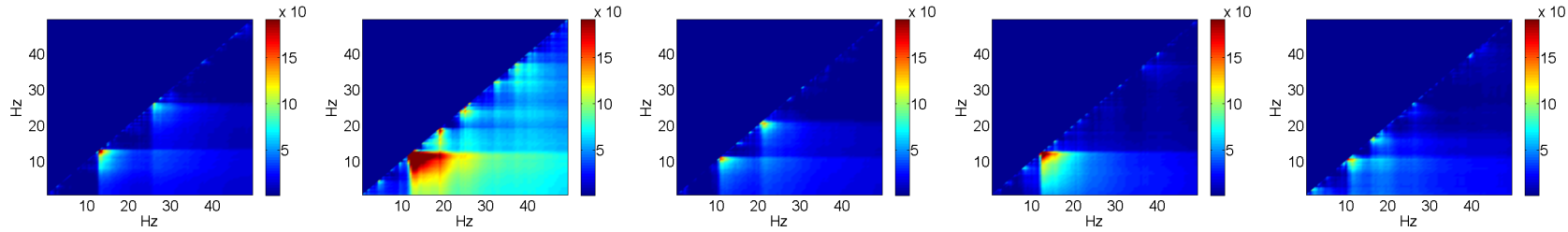

Figure 2: HSIC, encoded by the colour value for different frequency bands (axes correspond to upper and lower cutoff frequencies). The figures, left to right, top to bottom correspond to subjects 'aa', 'al', 'av', 'aw' and 'ay'.

SIC was used to select the top 5 discriminative frequency components based on the power spectrum. The 5 selected frequencies and their 4 nearest neighbours were used to reconstruct the temporal signals (with all other Fourier coefficients eliminated). The result was then passed to a normal CSP method (Dornhege et al., 2004) for feature extraction, and then classified using a linear SVM.

We compared automatic filtering using BAHSIC to other filtering approaches: normal CSP method with manual filtering $(8-40 \mathrm{~Hz})$, the CSSP method (Lemm et al., 2005), and the CSSSP method (Dornhege et al., 2006). All results presented in Table 2 are obtained using $50 \times 2$-fold cross-validation. Our method is very competitive and obtains the first and second place for 4 of the 5 subjects. While the CSSP and the CSSSP methods are specialised embedded methods (w.r.t. the CSP method) for frequency selection on BCI data, our method is entirely generic: BAHSIC decouples feature selection from CSP.

In Figure 2, we use HSIC to visualise the responsiveness of different frequency bands to motor imagination. The horizontal and the vertical axes in each subfigure represent the lower and upper bounds for a frequency band, respectively. HSIC is computed for each of these bands. Dornhege et al. (2006) report that the $\mu$ rhythm (approx. $12 \mathrm{~Hz}$ ) of EEG is most responsive to motor imagination, and that the $\beta$ rhythm (approx. 22 $\mathrm{Hz}$ ) is also responsive. We expect that HSIC will create a strong peak at the $\mu$ rhythm and a weaker peak at the $\beta$ rhythm, and the absence of other responsive frequency components will create block patterns. Both predictions are confirmed in Figure 2. Furthermore, the large area of the red region for subject 'al' indicates good responsiveness of his $\mu$ rhythm. This also corresponds well with the lowest classification er- 
ror obtained for him in Table 2 .

\section{Conclusion}

This paper proposes a backward elimination procedure for feature selection using the Hilbert-Schmidt Independence Criterion (HSIC). The idea behind the resulting algorithm, BAHSIC, is to choose the feature subset that maximises the dependence between the data and labels. With this interpretation, BAHSIC provides a unified feature selection framework for any form of supervised learning. The absence of bias and good convergence properties of the empirical HSIC estimate provide a strong theoretical justification for using HSIC in this context. Although BAHSIC is a filter method, it still demonstrates good performance compared with more specialised methods in both artificial and real world data. It is also very competitive in terms of runtime performance. ${ }^{6}$

Acknowledgments NICTA is funded through the Australian Government's Baking Australia's Ability initiative, in part through the ARC.This research was supported by the Pascal Network (IST-2002-506778).

Appendix: proof of Theorem 3 We first relate a biased estimator of HSIC to the biased estimator of MMD. The former is given by

$$
\frac{1}{(m-1)^{2}} \operatorname{tr} \mathbf{K H L H} \text { where } \mathbf{H}=\mathbf{I}-m^{-1} \mathbf{1} \mathbf{1}^{\top}
$$

and the bias is bounded by $O\left(m^{-1}\right)$, as shown by Gretton et al. (2005). An estimator of MMD with bias $O\left(m^{-1}\right)$ is

$$
\begin{aligned}
\operatorname{MMD}[\mathcal{F}, Z]= & \frac{1}{m_{+}^{2}} \sum_{i, j}^{m_{+}} k\left(\mathbf{x}_{i}, \mathbf{x}_{j}\right)+\frac{1}{m_{-}^{2}} \sum_{i, j}^{m_{-}} k\left(\mathbf{x}_{i}, \mathbf{x}_{j}\right) \\
& -\frac{2}{m_{+} m_{-}} \sum_{i}^{m_{+}} \sum_{j}^{m_{-}} k\left(\mathbf{x}_{i}, \mathbf{x}_{j}\right)=\operatorname{tr} \mathbf{K} \mathbf{L} .
\end{aligned}
$$

If we choose $l\left(y, y^{\prime}\right)=\rho(y) \rho\left(y^{\prime}\right)$ with $\rho(1)=m_{+}^{-1}$ and $\rho(-1)=m_{-}^{-1}$, we can see $\mathbf{L} \mathbf{1}=0$. In this case $\operatorname{tr} \mathbf{K} \mathbf{H} \mathbf{L} \mathbf{H}=\operatorname{tr} \mathbf{K} \mathbf{L}$, which shows that the biased estimators of MMD and HSIC are identical up to a constant factor. Since the bias of $\operatorname{tr} \mathbf{K} \mathbf{H L ~} \mathbf{H}$ is $O\left(\mathrm{~m}^{-1}\right)$, this implies the same bias for the MMD estimate.

To see the same result for Kernel Target Alignment, note that for equal class size the normalisations with regard to $m_{+}$and $m_{-}$become irrelevant, which yields the corresponding MMD term.

\footnotetext{
${ }^{6}$ Code is freely available as part of the Elefant package at http://elefant.developer.nicta.com.au.
}

\section{References}

Baker, C. (1973). Joint measures and cross-covariance operators. Transactions of the American Mathematical Society, 186, 273-289.

Borgwardt, K. M., Gretton, A., Rasch, M. J., Kriegel, H.P., Schölkopf, B., \& Smola, A. J. (2006). Integrating structured biological data by kernel maximum mean discrepancy. Bioinformatics (ISMB), 22(14), e49-e57.

Cristianini, N., Kandola, J., Elisseeff, A., \& Shawe-Taylor, J. (2003). On optimizing kernel alignment. Tech. rep., UC Davis Department of Statistics.

Dornhege, G., Blankertz, B., Curio, G., \& Müller, K. (2004). Boosting bit rates in non-invasive EEG singletrial classifications by feature combination and multiclass paradigms. IEEE Trans. Biomed. Eng., 51, 9931002 .

Dornhege, G., Blankertz, B., Krauledat, M., Losch, F., Curio, G., \& Müller, K. (2006). Optimizing spatio-temporal filters for improving BCI. In NIPS, vol. 18.

Fukumizu, K., Bach, F. R., \& Jordan, M. I. (2004). Dimensionality reduction for supervised learning with reproducing kernel hilbert spaces. JMLR, 5, 73-99.

Gretton, A., Bousquet, O., Smola, A., \& Schölkopf, B. (2005). Measuring statistical dependence with HilbertSchmidt norms. In $A L T, 63-78$.

Guyon, I., \& Elisseeff, A. (2003). An introduction to variable and feature selection. Journal of Machine Learning Research, 3, 1157-1182.

Guyon, I., Weston, J., Barnhill, S., \& Vapnik, V. (2002). Gene selection for cancer classification using support vector machines. Machine Learning, 46, 389-422.

Kira, K., \& Rendell, L. (1992). A practical approach to feature selection. In Proc. 9th Intl. Workshop on Machine Learning, 249-256.

Koller, D., \& Sahami, M. (1996). Toward optimal feature selection. In $I C M L, 284-292$.

Lemm, S., Blankertz, B., Curio, G., \& Mülller, K.-R. (2005). Spatio-spectral filters for improving the classification of single trial EEG. IEEE Trans. Biomed. Eng., 52, 1541-1548.

Nemenman, I., Shafee, F., \& Bialek, W. (2002). Entropy and inference, revisited. In NIPS, vol. 14.

Neumann, J., Schnörr, C., \& Steidl, G. (2005). Combined SVM-based feature selection and classification. Machine Learning, 61, 129-150.

Schölkopf, B., \& Smola, A. (2002). Learning with Kernels. Cambridge, MA: MIT Press.

Serfling, R. (1980). Approximation Theorems of Mathematical Statistics. New York: Wiley.

Song, L., Smola, A., Gretton, A., Borgwardt, K., \& Bedo, J. (2007). Feature selection for supervised learning using Hilbert-Schmidt Independence Criterion. Tech. rep., NICTA, ANU.

Steinwart, I. (2002). On the influence of the kernel on the consistency of svms. JMLR, 2, 67-93.

Weston, J., Elisseeff, A., Schölkopf, B., \& Tipping, M. (2003). Use of zero-norm with linear models and kernel methods. JMLR, 3, 1439-1461.

Weston, J., Mukherjee, S., Chapelle, O., Pontil, M., Poggio, T., \& Vapnik, V. (2000). Feature selection for SVMs. In NIPS, vol. 13.

Zaffalon, M., \& Hutter, M. (2002). Robust feature selection using distributions of mutual information. In $U A I$. 